\title{
Phase behaviour of cellulose nanocrystal dispersion in aqueous sulphuric acid and development of an energy efficient separation technique for the acid-cellulose nanocrystal system
}

\author{
Soon Yee Liew, ${ }^{1, a}$ Wim Thielemans ${ }^{2, b}$ and Buddhika Hewakandamby ${ }^{1, c}$ \\ ${ }^{1}$ Division of Manufacturing and Process Technologies, Faculty of Engineering, \\ University of Nottingham, Nottingham NG7 2RD \\ ${ }^{2}$ Renewable Materials and Nanotechnology Research Group, \\ Department of Chemical Engineering, KU Leuven, Campus Kortrijk, \\ Etienne Sabbelaan 53, 8500 Kortrijk Belgium \\ a soon.liew@nottingham.ac.uk, ${ }^{b}$ wim.thielemans@kuleuven.be, \\ ${ }^{\mathrm{c}}$ buddhika.hewakandamby@nottingham.ac.uk
}

Keywords: Cellulose nanocrystals, sulphuric acid, nanoparticle suspension, phase behavior, interaction energy, nanoparticle separation, process energy efficiency, cellulose nanocrystals recovery, acid recovery.

\begin{abstract}
In this paper, the phase behaviour of a cellulose nanocrystal (CNCs) dispersion in sulphuric acid solutions was investigated, aimed at the development of an energy efficient separation method for this mixture. The system in consideration was a mixture of $30 \mathrm{wt} \%$ aqueous sulphuric acid $\left(\rho_{1}=1219 \mathrm{~kg} / \mathrm{m}^{3}\right)$ containing $12.6 \mathrm{mg} / \mathrm{ml}$ of cellulose nanocrystals (CNCs) $\left(\rho_{\mathrm{s}}=1590 \mathrm{~kg} / \mathrm{m}^{3}\right.$, volume fraction of CNCs less than $\left.1 \%\right)$. This volume filling mixture was obtained directly from a CNC extraction process, as obtained after the hydrolysis of cotton using $64 \mathrm{wt} \%$ sulphuric acid at $c a .45^{\circ} \mathrm{C}$ for 45 minutes (this condition was required for the extraction of CNCs from cotton) followed by quenching the hydrolysis with water. The CNCs form the desired product and need to be separated from the acid that can then be recycled. Conventionally this separation has been difficult and requires a large input of energy. This work addresses this problem by investigating into the phase behaviour and physicochemical and hydrodynamic character of this mixture. This understanding led to the development of a very energy efficient separation mechanism for this mixture, which is 5 orders of magnitude more energy efficient than the most widely used centrifugation systems.
\end{abstract}

\section{Introduction}

The research on and use of advanced materials derived from renewable resources has increased significantly over recent years, encouraged by an increasing environmental consciousness of the general population. As the most abundant renewable material on Earth, cellulose and its derivatives have been studied for use in many different areas. Among these, a very actively researched area focuses on the fundamentals and applications of cellulose nanocrystals (CNCs) [1-3]. CNCs are the elementary crystallites within native cellulose materials, and can be extracted from the native cellulose materials by selectively removing the amorphous cellulose sections. As monocrystalline rigid rods, CNCs exhibit a remarkable mechanical strength and for this reason attracts a huge interest in structural reinforcement applications such as polymer composites [4-10]. The high aspect ratio of CNCs also means that they easily form percolated network structures and for this reason CNCs have been used for making aerogels [11], and 
membranes. As CNCs in their unmodified form are biocompatible, they could be used for the development of biomaterials and biocomposites [3,12,13]. Other applications that utilise CNCs for their unique physical and chemical characteristics are for example sensor materials [14-17] and as supercapacitor electrode support material [18-22].

Typical sizes of CNCs are in the range of 6-20 nm cross section and 100-400 $\mathrm{nm}$ in length [23]. The dimensional variation arises from the different cellulose sources from which the CNCs are extracted and from the extraction conditions. The size and the shape of CNCs, while opening up many fascinating aspects that enable very interesting applications, they could also pose challenges in their extraction process on an industrial scale. The preparation of CNCs involves the controlled hydrolysis (35-45 minutes) of native cellulose in highly concentrated acid, followed by separation of the CNCs from the acid. The separation of CNCs from the acid is a very difficult processing step. At the laboratory scale, the separation-purification is typically realised by successive cycles of dilution and centrifugation [6,24-26]. Due to the small size of CNCs, settling velocities are relatively small. This means to separate the CNCs, centrifugation is not particularly effective unless at high-speed $(>10000 \mathrm{rpm})[6,26,27]$. In addition, when carrying out the acid hydrolysis with sulphuric acid, a small amount of sulphate half-esters are grafted on the CNC surface. As more acid is removed, they will deprotonate and provide electrostatic stabilisation of the CNCs making sedimentation by centrifugation almost impossible. While this centrifugation process is convenient at laboratory scale, it is foreseeable that, discounting the difficulty faced with developing a big centrifuge to run at high speed, the high energy cost alone would forbid this operation for bulk materials production. Thus, in order to promote energy- and cost-effective industrial scale production of CNCs, innovation in process technologies for the acid-CNC separation is required.

In our previous work, we showed that mixtures of CNCs in sulphuric acid (acid concentration ranging from $5 \mathrm{wt} \%$ to $30 \mathrm{wt} \%$ ) with a solid content above $12.6 \mathrm{mg} / \mathrm{ml}$ can be separated readily after they were shaken in a closed vessel and left to stand [28]. Through shaking, a large number of small air bubbles were introduced into the mixture and the separation took place when CNCs appear to float with the bubbles to leave at the bottom of the container a clear solution of sulphuric acid. It was thought that when the air bubbles rise through the shaken mixture, the shear forces exerted on the mixture creates denser flocs of CNCs. The result of the two effects combined was the observed flotation of CNCs to leave a clear acid solution at the bottom of the container. This simple process demonstrated an energy efficient separation of CNCs from the acid and can potentially have a big impact on the industrial scale production of these materials.

The present study further develops this idea that the acid-CNC separation can be performed with very little energy input, such as by manual shaking and followed by a rest period [28], as has been demonstrated previously. To achieve this, it is necessary to understand the phase behaviour of CNCs in acidic mixtures (30 wt \% sulphuric acid). For example, how a volume filling gel [29] forms when the CNC content increases to $12.6 \mathrm{mg} / \mathrm{ml}$ so that this mixture remained stable for very long time [28]. The interaction energy between CNCs as a function of the interparticle distance in the acidic mixture was evaluated. The result suggested that despite only occupying a very low volume fraction, the CNC structures can be selfsupporting within the acidic environment and it was on this basis that the volume filling gel was formed. However, the calculations also show that it is favourable for the CNCs structures to become further compacted to a higher density structure. The strategy employed to separate CNCs from the acid environment was therefore based on an interfacial shearing/pressing mechanism, aiming at re-organising the $\mathrm{CNC}$ gel structure into a more compact state. Thus starting from an acid-CNC mixture that is initially homogeneous, the liquid acid could be pressed out of the CNC gel structure. Using a $2 \mathrm{~L}$ and a $5 \mathrm{~L}$ prototype, a $65 \%$ liquid acid removal rate was achieved in one press, which took place over 8 and 12 hours, respectively. 
The evaluation of the energy input required for this separation, on a volume basis, shows that it was 5 orders of magnitude more efficient than the laboratory centrifuge for the same separation efficiency. Thus, we believe that this work can be adopted into future large scale $\mathrm{CNC}$ production, towards a more energy efficient and sustainable manufacturing process.

\section{Materials and methods}

Preparation of acid-CNC mixture. The cellulose source was cotton wool purchased from Scientific Laboratory Supplies. Sulphuric acid (ACS reagent grade) was purchased from Sigma-Aldrich. The method of cotton hydrolysis follows the method described by Revol et al. [30]. Typically, $400 \mathrm{ml}$ of $64 \mathrm{wt} \% \mathrm{H}_{2} \mathrm{SO}_{4}$ was used for $45.7 \mathrm{~g}$ of cotton wool ( $8.75 \mathrm{ml}$ of $64 \mathrm{wt} \%$ acid per gram of cotton) at $45^{\circ} \mathrm{C}$ for 45 minutes. After 45 minutesthis mixture was diluted with $700 \mathrm{ml}$ of water to produce a resulting mixture with an acid concentration of $c a .30 \mathrm{wt} \%$ and containing $12.6 \mathrm{mg} / \mathrm{ml} \mathrm{CNC} \mathrm{(denoted} \mathrm{the} \mathrm{acid-CNC} \mathrm{mixture} \mathrm{throughout} \mathrm{the} \mathrm{rest} \mathrm{of} \mathrm{this} \mathrm{paper).}$ This acid-CNC mixture was allowed to cool to room temperature and used without further manipulations.

Press Separator prototype. The separator prototype was a vertical press using a $2 \mathrm{~L}$ (Figure 5) and a $5 \mathrm{~L}$ (Figure 9) beaker, similar to a household coffee press. The press was driven by a 24 Volt DC electric motor using 0.01 Amp current. The current was supplied by either manual pulsing or continuously. The press comprised of a piston made of a perforated grey PVC plate, 3 layers of polypropylene net and a $4 \mathrm{~cm}$ thick bed of $2 \mathrm{~mm}$ average diameter polystyrene (PS) foam beads. The bed of PS beads provides a high surface area for CNC attachment and for delivering the pressure onto the CNC structure only. As the PS beads have a very low density and float in the mixture, the beads push against the piston and this was convenient for maintaining a consistent downward pressing action. The tendency of the PS beads to float was also very useful for their recovery after a separation cycle was completed. By pressing downwards, the volume filling CNCs (details vide infra) were compacted at the bottom and, as a result, the liquid acid was pressed out and accumulated as the top layer. The optimum pressing speed was found to be $1 \mathrm{~cm}$ per hour. This was experimentally determined and details will be discussed in the results and discussion section.

\section{Theoretical background}

The starting point of this discussion is the acid-CNC mixture, which is a suspension of CNCs in $30 \mathrm{wt} \% \mathrm{H}_{2} \mathrm{SO}_{4}$. The solid content in this suspension is $12.6 \mathrm{mg} / \mathrm{ml}$. The CNC suspension is stable, and the CNCs fill the total mixture volume. Gravitational settling was not seen to occur in this mixture for up to 2 years (Figure 2). When this suspension was shaken in a closed container and left to stand, separation occurs as described by us previously [28]. To understand this behaviour, a theoretical investigation was carried out on the CNC dispersion stability and interaction energy between CNCs.

Dispersion stability. The CNCs extracted by sulphuric acid hydrolysis display a negative surface charge when dispersed in water. This is due to the conversion of some of the primary hydroxyls to sulphate halfesters which deprotonate in $\mathrm{pH}$-neutral water. The dispersion stability of individualized CNCs in $30 \mathrm{wt} \%$ sulphuric acid can be characterised using the zeta potential of the CNCs in suspension. From the definitions of surface charge, the zeta potential is given by:

$\zeta=\frac{q_{s \lambda_{D}}}{\epsilon}$ 
where $\zeta$ is the zeta potential $(\mathrm{V}), \mathrm{q}_{\mathrm{s}}$ is the surface charge $\left(\mathrm{C} \mathrm{m}^{-2}\right), \epsilon$ is the permittivity of the solvent, $30 \mathrm{wt} \%$ sulphuric acid $\left(84 \times 8.854 \times 10^{-12} \mathrm{C} \mathrm{V}^{-1} \mathrm{~m}^{-1}\right), \lambda_{\mathrm{D}}$ is the Debye length $(\mathrm{m})$ given by $9.61 \times 10^{-9} /\left(\mathrm{z}^{2} \mathrm{c}\right)^{1 / 2}$ where $\mathrm{z}$ is the charge number and $\mathrm{c}$ is the concentration in $\mathrm{mol} \mathrm{m}^{-3}$.

Using conductimetric titration, the CNC surface charge was determined to be $0.1 \mathrm{mmol} \mathrm{g}^{-1}$. Incorporating the specific surface of individual CNCs $\left(440 \mathrm{~m}^{2} \mathrm{~g}^{-1}\right.$ based on their average dimensions), the surface charge of CNCs is $0.02173 \mathrm{C} \mathrm{m}^{-2}$. In $30 \mathrm{wt} \%$ acid, the Debye length is around $1 \dot{\mathrm{A}}$. Thus the zeta potential of CNCs in $30 \mathrm{wt} \%$ sulphuric acid is calculated as 3 $\mathrm{mV}$. This result means that the $\mathrm{CNC}$ dispersion in $30 \mathrm{wt} \%$ sulphuric acid is not electrostatically stabilised, but will exist as a suspended solid. This result is consistent with the observed behaviour of the acid-CNC mixture in this work.

Interaction energy between $\mathrm{CNC}$ particles. The calculation of interaction energy between CNC particles can be carried out using the procedures described in Buining and co-workers (originally derived by Sparnaay [31]) for two cylinder-shaped colloidal particles [29]. For simplicity, the interaction energy calculation was carried out only for the interaction at parallel orientation of the nanoparticles. The energy calculation for interaction in a crossed orientation was not carried out for the reason that it was significantly more complex and laborious, but the end result was consistent with the parallel-oriented interaction [29]. The repulsive energy due to parallel double-layer overlap for cylinders as a function of their surface to surface distance, $H_{0}$, is [29]

$V_{R}=64 \pi^{\frac{1}{2}} n k T \gamma^{2} L \frac{(\kappa a)^{\frac{1}{2}}}{\kappa^{2}} \exp \left(-\kappa H_{0}\right)$

with

$\gamma=\tanh \left(\frac{e \zeta}{4 k T}\right)$

In these equations, $n$ is the number density of ions in the solution, $k$ is the Boltzmann constant, $T$ is temperature $(\mathrm{K}), \kappa$ is the reciprocal Debye length, $a$ is the cylinder radius (CNC average width, $6 \mathrm{~nm}$ ), L is the particle length (CNC average length, $150 \mathrm{~nm}$ ), $\zeta$ is the zeta potential $(3 \mathrm{mV})$, and $e$ is the elementary electric charge.

For parallel cylinders, the van der Waals attractive energy is

$V_{A}=-\frac{3 \pi}{8} A \frac{L}{a} a^{5} U_{5}$

with $A$ the Hamaker constant, which is $8 \times 10-{ }^{21} \mathrm{~J}$ for cellulose water systems, [32] and $U_{5}$ is given by a series equation (Buining and co-workers [29] only considered the first five terms):

$U_{5}=\frac{1}{R^{5}}\left\{1+6.25\left(\frac{a}{R}\right)^{2}+31.9\left(\frac{a}{R}\right)^{4}+150.7\left(\frac{a}{R}\right)^{6}+684\left(\frac{a}{R}\right)^{8}+2200\left(\frac{a}{R}\right)^{10}+\cdots\right\}$

where $R$ is the distance between the cylinder axes, taken as $H_{0}+6 \mathrm{~nm}$ (where half the CNC average width is $3 \mathrm{~nm}$ ). The net interaction energy between the individual CNCs in the acid, $V$, is $V_{A}+V_{R}$. Figure 1 shows the plot of $V$ in units of $\mathrm{kT}$ as a function of distance $H_{0}$. 


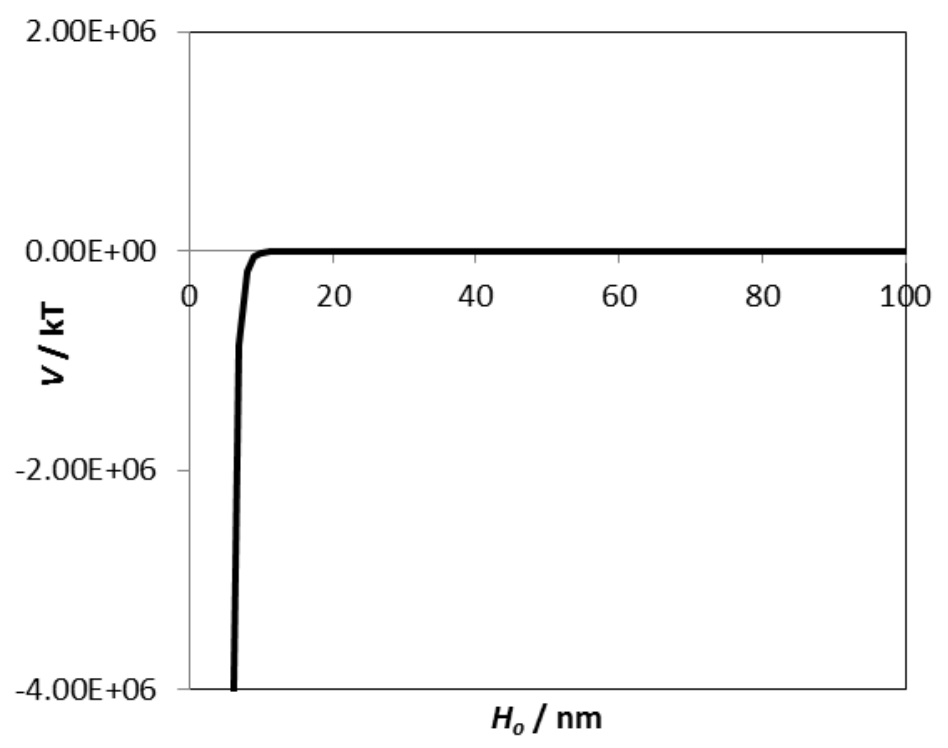

Figure 1. Interaction energy between 2 parallel oriented $\mathrm{CNC}$ particles in $30 \mathrm{wt} \%$ sulphuric acid.

Figure 1 shows that the interaction energy between $2 \mathrm{CNC}$ particles at any distance is negative and approaches zero at larger distances. The repulsive energy, $V_{R}$, calculated for all distances was found to be insignificant compared to the attractive energy. For this reason, the repulsive regime which is common for colloidal particles at small interparticle distance and lower electrolyte concentration was not observed in Figure 1. Table 1 shows some of the $V$ values at a few representative distances.

Table 1. Total interaction energy, $V$, between 2 parallel oriented $\mathrm{CNC}$ particles at different distances $H_{0}$.

\begin{tabular}{|c|c|}
\hline $\begin{array}{ll}\text { Distance, } & H_{0} \\
(\mathrm{~nm})\end{array}$ & $V(\mathrm{kT})$ \\
\hline 5 & -37600000 \\
\hline 10 & -13500 \\
\hline 15 & -140 \\
\hline 20 & -8.36 \\
\hline 25 & -1.38 \\
\hline 50 & -0.0190 \\
\hline
\end{tabular}

When $2 \mathrm{CNC}$ particles are $50 \mathrm{~nm}$ apart, the interaction energy is $-0.019 \mathrm{kT}$. While this means that they are attracted to one another, this small force is easily cancelled by Brownian motion of the surrounding molecules and the CNC particles themselves, which averages about $1 \mathrm{kT}$. When $2 \mathrm{CNC}$ particles are at $25 \mathrm{~nm}$ apart, the interaction energy becomes significant, which is $-1.38 \mathrm{kT}$. This means that the interaction energy (attractive) between the CNC particles will not be cancelled out by the thermal motion of the surrounding molecules. The interaction energy becomes significantly higher when CNC particles are less than $20 \mathrm{~nm}$ apart (-8.36 kT and higher) where the attractive forces are significantly higher than Brownian motions and become dominant.

Distance between particles. As presented above, the interaction energy between $2 \mathrm{CNC}$ particles is a function of distance between the 2 particles. Thus knowing the distance between 
CNC particles in a particular suspension would give an indication of the strength of the interaction. Given the solids content of CNCs in the acid suspension $\left(12.6 \mathrm{mg} / \mathrm{cm}^{3}\right)$, the number of individual CNCs in $1 \mathrm{~cm}^{3}$ of suspension can be determined, knowing the mass for a mole of CNC is $4.98 \times 10^{6} \mathrm{~g}$ (from particles of dimensions $150 \mathrm{~nm} \times 6 \mathrm{~nm} \times 6 \mathrm{~nm}$ ). The average distance between $\mathrm{CNC}$ particles is then calculated to be $c a .48 .7 \mathrm{~nm}$. Meanwhile, the volume fraction of the $\mathrm{CNC}$ particles in the mixture is $0.8 \%$. These calculations mean that the CNCs only occupy a small fraction of the total volume of the suspension $\left(0.008 \mathrm{~cm}^{3}\right.$ out of 1 $\mathrm{cm}^{3}$ ). Yet that the CNC can fill the whole mixture volume at such a low volume fraction and remain very stable suggest that the CNC particles interact strongly with each other. Knowing the average distance between the $\mathrm{CNC}$ particles will allow the construction of a picture representing the acid-CNC mixture at the nanoscale in the next section. This will visually aid the understanding of the volume-filling phase behaviour of CNCs in this acid-CNC mixture.

\section{Results and discussion}

Interaction energy and $\mathrm{CNC}$ arrangement in the acid-CNC mixture. Acid-CNC mixtures $(12.6 \mathrm{mg} / \mathrm{ml} \mathrm{CNC}$ in $30 \mathrm{wt} \%$ sulphuric acid) as prepared and left on the bench for for respectively 3 days and 2 years are shown in Figure 2. It can be seen that the CNCs in the mixture occupied the whole volume of the mixture and did not settle out even at long times. Such mixtures are generally referred to as volume-filling mixtures or volume-filling gels.[29]

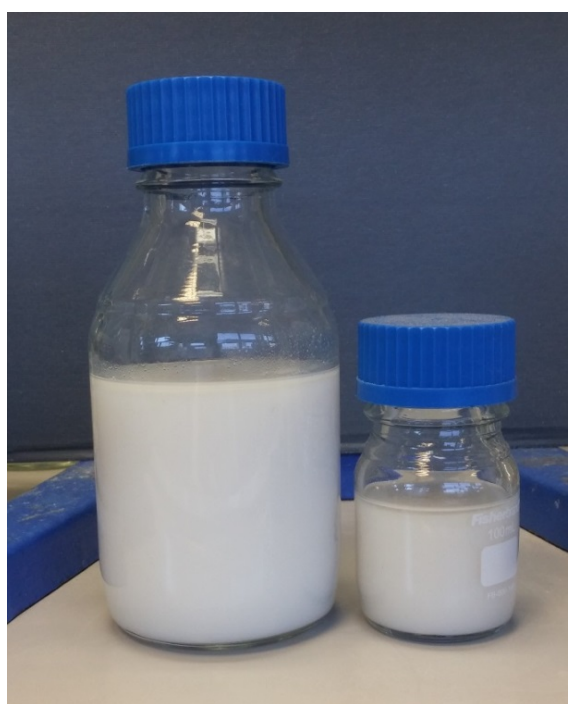

Figure 2. Acid-CNC mixtures of $12.6 \mathrm{mg} / \mathrm{ml} \mathrm{CNC} \mathrm{in} 30 \mathrm{wt} \%$ sulphuric acid (+ hydrolysis products) as prepared and left on bench for (left) 3 days and (right) 2 years.

A 2D representation for some randomly oriented CNC particles in the acid-CNC mixture is shown in Figure 3, which was drawn with a $48.7 \mathrm{~nm}$ average distance between the centres of the $\mathrm{CNC}$ particles relative to the CNC thickness. In this 2D representation, it can be seen that while the centres of individual $\mathrm{CNC}$ rods are $48.7 \mathrm{~nm}$ apart from the centres of the nearest neighbour $\mathrm{CNC}$ rods on average, these $\mathrm{CNC}$ particles may also be in contact with other CNCs at other points along the length of the CNCs. This raises several points for discussion. Firstly, the contact between CNC particles is energetically favourable as predicted by the interaction energy calculations, and especially when the particles approach each other, below a distance of $20 \mathrm{~nm}$, the attractive interaction energy increases exponentially with decreasing distance. Our calculation above shows that at a $5 \mathrm{~nm}$ distance, $V$ is $-3.8 \times 10^{8} \mathrm{kT}$. The fact that the CNCs do not contact one another in parallel orientation means that the calculations present an 
overestimation, as they were carried out for parallel orientation only. Nevertheless, the energy of interaction, both for a parallel and crossed orientation, follows the same trend. Estimating the interaction energy value at these points of contact, detailed calculations in previous work [29] showed the crossed configuration interaction energy to be roughly 2 orders of magnitude lower than the parallel interaction value, which is still rather significant at small separation distances. Moreover, the repulsive interactions between the CNC particles are negligible in the system. This means that the CNCs in the acidic suspension are able to form a network structure that fills the mixture volume, similar to the illustration in Figure 3. When the CNC content is lower, for example at $10 \mathrm{mg} / \mathrm{ml}$, the average particle distance calculated was $53 \mathrm{~nm}$ and when a similar drawing to Figure 3 was created (not shown), the number of contact points were less and assembling a completely interconnected network showed significantly larger void areas where the CNC structures might collapse. A corollary was that in the $30 \mathrm{wt} \%$ sulphuric acid environment, a CNC content of $12.6 \mathrm{mg} / \mathrm{ml}$ was probably just above the percolation threshold that the volume filling behaviour takes place. This can explain the observation that such mixtures remain stable and do not separate over a long time, i.e. for at least 2 years.

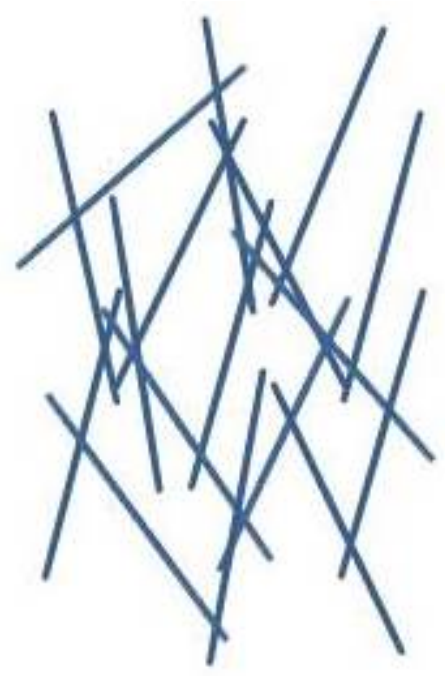

Figure 3. A possible arrangement of CNC particles (rods of $6 \mathrm{~nm} \times 6 \mathrm{~nm} \times 150 \mathrm{~nm}$ ) in the acid-CNC mixture, containing $12.6 \mathrm{mg} / \mathrm{ml}$ CNCs in $30 \mathrm{wt} \%$ sulphuric acid. The average distances between particles were $48.7 \mathrm{~nm}$. This drawing is scaled by the CNC rods which are $150 \mathrm{~nm}$ in length.

The proposed method in this work, to separate the acid and the CNCs from the acid-CNC mixture was so that the CNCs were rearranged, in this case being compressed to become a network of higher density (Figure 4). As shown by the energy calculation, the CNCs favour being closer to one another. When the $\mathrm{CNC}$ structure was being compacted, liquid acid necessarily needs to counter diffuse out to make space for more CNC to occupy the same volume. In other words, the liquid acid will be pressed out of the porous CNC network as the $\mathrm{CNC}$ network is being compressed. In this acidic environment, the CNCs can approach external surfaces very closely, which was another implication of the interaction energy calculation. Utilising this behaviour, the pressure can be delivered to the CNC structure by a moving external surface. It is worthy to note that when the CNC surfaces are not charged (as in this work, due to the protonation of the $\mathrm{CNC}$ surface sulphonate groups), they can stabilise the oil/water interface in so-called Pickering emulsions [33,34]. Therefore, the stability of the $\mathrm{CNC}$ structures at the surface of external bodies was utilised in this work for the separation process development. 


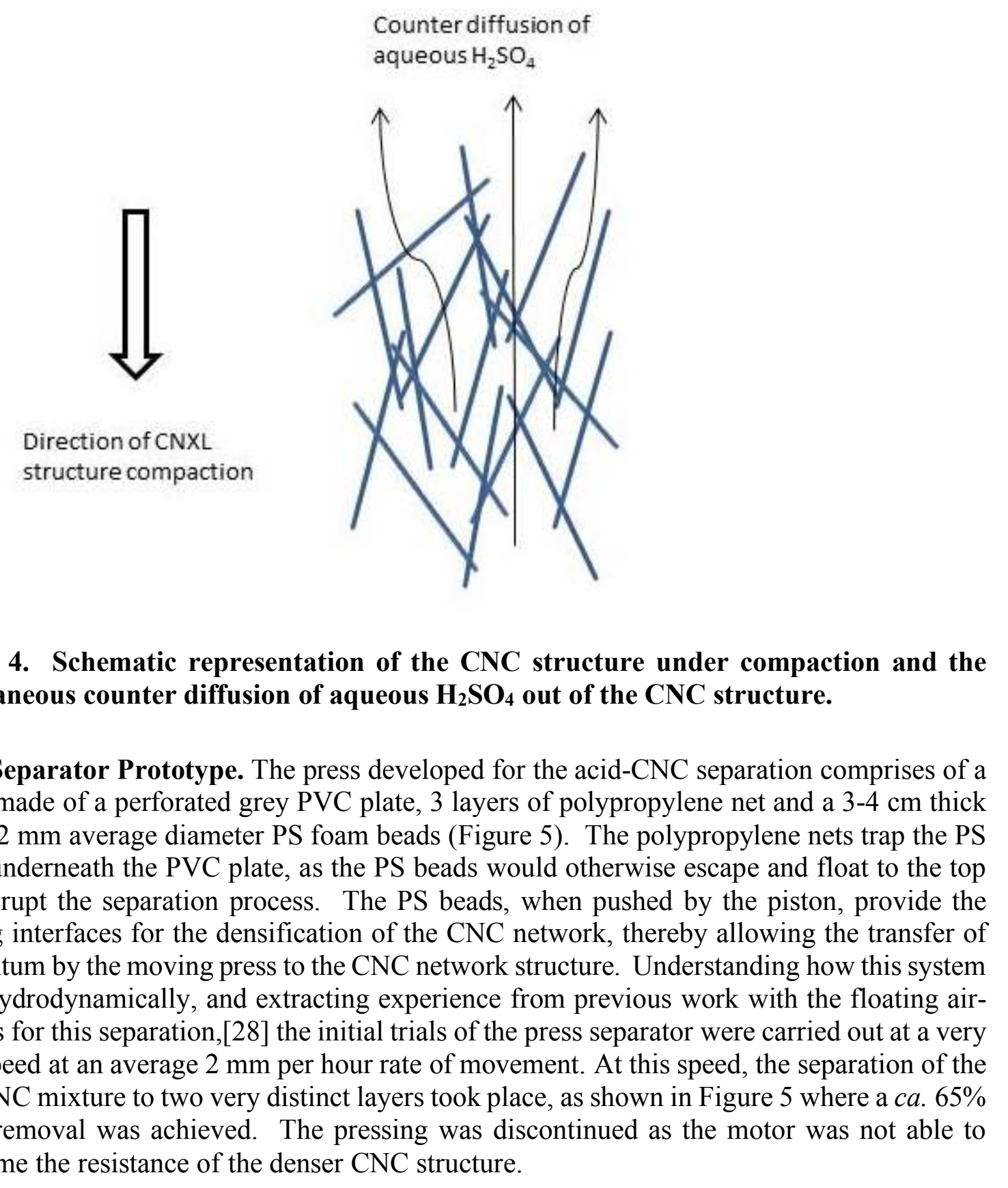

Figure 4. Schematic representation of the CNC structure under compaction and the simultaneous counter diffusion of aqueous $\mathrm{H}_{2} \mathrm{SO}_{4}$ out of the $\mathrm{CNC}$ structure.

Press Separator Prototype. The press developed for the acid-CNC separation comprises of a piston made of a perforated grey PVC plate, 3 layers of polypropylene net and a 3-4 cm thick bed of $2 \mathrm{~mm}$ average diameter PS foam beads (Figure 5). The polypropylene nets trap the PS beads underneath the PVC plate, as the PS beads would otherwise escape and float to the top and disrupt the separation process. The PS beads, when pushed by the piston, provide the moving interfaces for the densification of the $\mathrm{CNC}$ network, thereby allowing the transfer of momentum by the moving press to the $\mathrm{CNC}$ network structure. Understanding how this system work hydrodynamically, and extracting experience from previous work with the floating airbubbles for this separation, [28] the initial trials of the press separator were carried out at a very slow speed at an average $2 \mathrm{~mm}$ per hour rate of movement. At this speed, the separation of the acid-CNC mixture to two very distinct layers took place, as shown in Figure 5 where a $c a .65 \%$ liquid removal was achieved. The pressing was discontinued as the motor was not able to overcome the resistance of the denser $\mathrm{CNC}$ structure. 


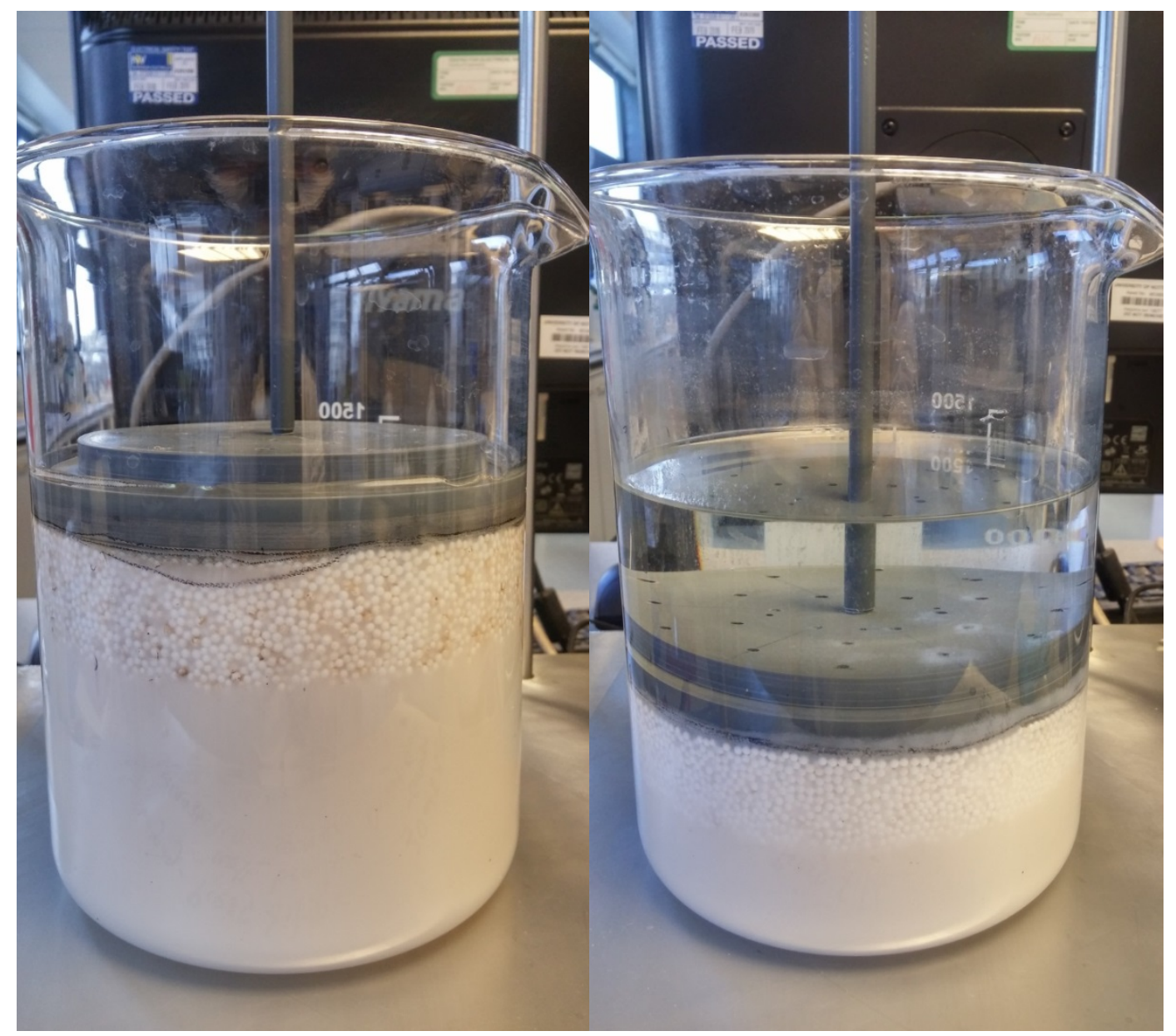

Figure 5. Press separator prototype for the acid-CNC separation in a $2 \mathrm{~L}$ sized beaker. (Left) Before and (right) after the press separation.

When the CNCs were compacted to within $35 \%$ of the original volume, from $12.6 \mathrm{mg} / \mathrm{ml}$ to 36 $\mathrm{mg} / \mathrm{ml}$, they formed a very dense and thick paste at the bottom of the beaker. At this CNC content, the average distance between the individual CNC particle centres becomes $34.3 \mathrm{~nm}$. The possible arrangement of the CNCs in this mixture (2D) is shown in Figure 6. Compared to Figure 3, which was drawn for a $12.6 \mathrm{mg} / \mathrm{ml} \mathrm{CNC} \mathrm{content,} \mathrm{it} \mathrm{is} \mathrm{obvious} \mathrm{that} \mathrm{at} 36 \mathrm{mg} / \mathrm{ml}$ $\mathrm{CNC}$ content, as shown in Figure 6 had a denser structure, presenting a significantly larger number of contact points between the CNCs. To further compact the CNC beyond $36 \mathrm{mg} / \mathrm{ml}$ would require overcoming the attractive interactions between the $\mathrm{CNC}$ particles to form it into a new arrangement requiring a significantly higher amount of energy. In other words, the dense $\mathrm{CNC}$ structure resists further compaction. The small electric motor used to drive the press in this work did not have sufficient torque to overcome the resistance presented by the CNC structure and for this reason our experiments were typically stopped when 60-65\% liquid acid removal was achieved. Moreover, this was also the typical liquid removal rate achieved when the mixture was separated by centrifugation at $10000 \mathrm{rpm}$ for 10 minutes (which will be the basis for energy comparison) as shown in Figure 7. 


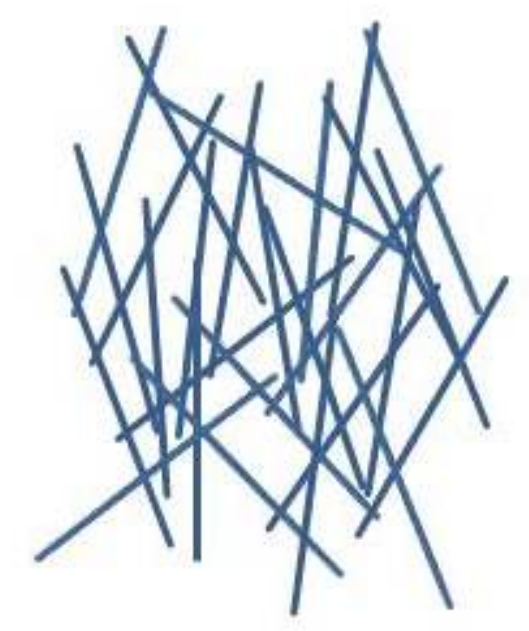

Figure 6. A possible arrangement of CNC particles (rods of $6 \mathrm{~nm} \times 6 \mathrm{~nm} \times 150 \mathrm{~nm}$ ) in the acid-CNC mixture, containing $36 \mathrm{mg} / \mathrm{ml}$ CNCs in $30 \mathrm{wt} \%$ sulphuric acid. The average distances between particles were $34.3 \mathrm{~nm}$. This drawing is scaled by the CNC rods which are $150 \mathrm{~nm}$ in length.

When the liquid acid was removed, the PS beads could be readily removed by flotation upon addition of water (Figure 8). Figure 8 also shows the dense CNC paste, which was compacted at the bottom of a $2 \mathrm{~L}$ beaker. This thick paste has very similar consistency to that obtained by centrifugation (Figure 7), which suggest a similar order of CNC packing between these samples. Of course, at a rate of $2 \mathrm{~mm}$ per hour, to complete the separation shown in Figure 3 required a long time, i.e. roughly 40 hours. Subsequently, attempts were made to operate the press at a higher speed to achieve the same separation results, therefore improving the overall effectiveness of this process. It was found that the same results can be achieved, operating at an average $1 \mathrm{~cm}$ per hour. At this speed, some CNCs escape the trap formed by the PS bed and remix downstream of the PVC piston, forming a layer of loosely packed sedimentation just above the PVC piston (Figure 9). More details on how the CNC escape takes place will be discussed in the next section. Nonetheless, at $1 \mathrm{~cm}$ per hour rate of the press movement, the amount of non-removed CNCs was not significant and as they always settle on top of the PVC piston, the clear liquid acid above can be removed without difficulty. The amount of escaped CNCs increases when speed of pressing increases beyond $1 \mathrm{~cm}$ per hour. Therefore, for this press separator design, the optimised speed was $1 \mathrm{~cm}$ per hour. 


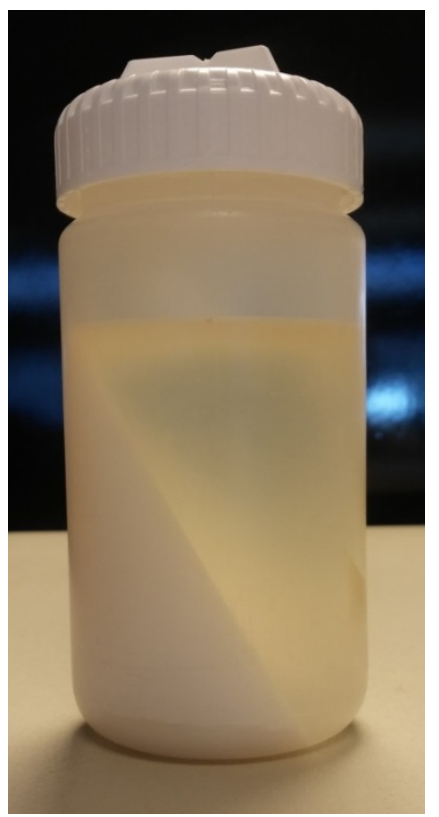

\section{Figure 7. Photograph image showing CNC sedimentation after subjecting a $250 \mathrm{ml}$ acid-CNC mixture of $12.6 \mathrm{mg} / \mathrm{ml} \mathrm{CNC}$ in $30 \mathrm{wt} \%$ sulphuric acid to centrifugation at $10000 \mathrm{rpm}$ for 10 minutes. Liquid removal rate was $C a .65 \%$.}

The escape of the CNCs from the PS bed can be explained as follows. When the CNC structure is being at very slow speed compacted, liquid acid necessarily counter diffuses out to make space for more CNCs to occupy the same volume (Figure 4). At a low operating speed, the liquid acid can very effectively diffuse out of the CNC structure under compaction, without causing disruption to the $\mathrm{CNC}$ structure. At a high speed however, this means that fast transport of liquid acid out of the CNC structure was also required. This becomes unfavourable past a certain point when the fast counter liquid transport disrupts the $\mathrm{CNC}$ structure. The fast liquid transport at hihgher pressing pseeds causes the CNCs at the edge close to the PVC piston perforations to thin out as a result of the shear-thinning due to particle alignment along the shear flow direction as has been demonstrated for fluid systems containing CNCs [35]. The aligned CNCs that will not be restricted by the gelled network will be able to flow with the liquid upwards to past the PVC piston. This process is further magnified by the discontinuous movement of the motor, as the jerking movement of the piston could also have caused breaking up of the CNC structure. In the process studied here, the loss of structure was obviously an edge effect as it did not affect the compaction of the CNC structure within the bulk mixture. This could be expected as the shear forces within the gaps between the PS beadsis expected to be significantly higher than below it where only the CNC-acid mixture exists. Therefore separation can still take place, although slightly less effective due to some CNCs escaping at the edge. The second explanation could be that at higher flow rates, the nature of the flow becomes advective and causes a wide disruption to the CNC structure, which may affect the whole mixture volume. In this case, the separation will not be possible as the advection would also cause mixing and this is not seen during the experiments. Further improvement on the pressing speed (separation speed) may be possible with a re-design of the press, but it is likely that there will be a hydrodynamic limit on the rate of this separation, based on the onset of advective fluid transport. 


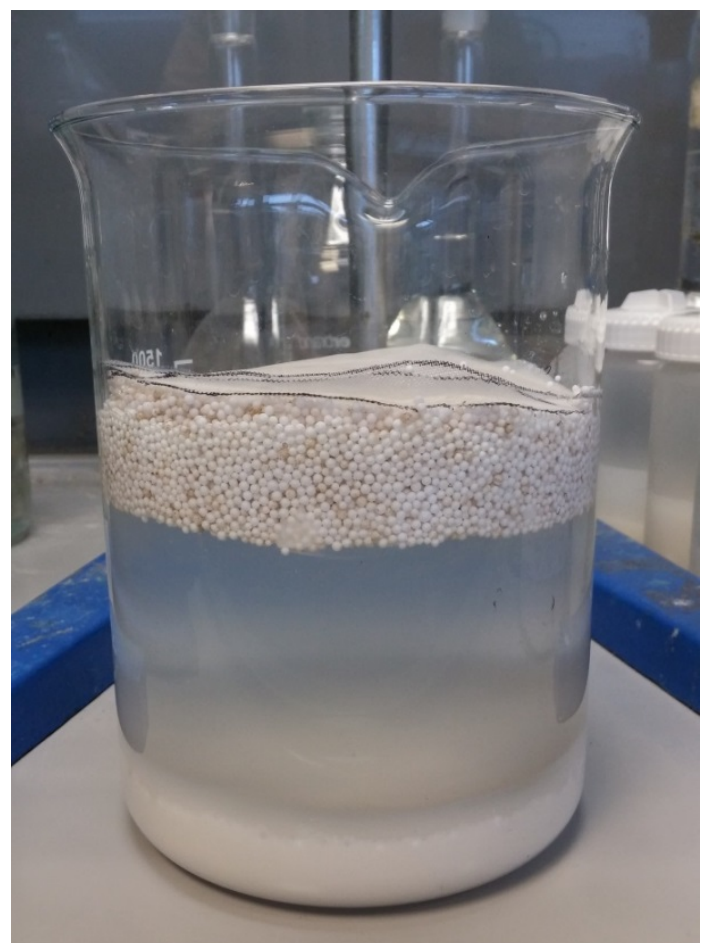

Figure 8. Recover of polystyrene beads after liquid acid removal as the beads float when water was added after the initial liquid acid was removed. Note the dense compacted CNC layer at the bottom of the beaker.

Energy Efficiency of the Press Separator. Further scale up of the press separator was carried out on a $5 \mathrm{~L}$ beaker. Figure 9 shows a completed separation ( $>65 \%$ liquid removal) of the acid$\mathrm{CNC}$ mixture by the press separator on a $5 \mathrm{~L}$ scale. One can see (Figure 9) that a small amount of CNCs have escaped the PS bed with the liquid flow and have settled above the PVC piston as described above. To achieve this separation, at $c a$. $65 \%$ liquid removal, the press travelled $12 \mathrm{~cm}$. The total energy was calculated using the equation $E=V i t$, where $V$ is the motor voltage (24 Volts), $i$ is the supply current (0.01 Amperes) and $t$ is the time required in seconds. In the experiment, as the current was supplied to the motor by pulsing, determining the total operation time of the motor was therefore estimated by the time required when the press was operated continuously. At continuous operation, the press required 30 seconds for $12 \mathrm{~cm}$ movement. Thus, the energy input for this separation of $3.6 \mathrm{~L}$ of acid-CNC mixture was $7.2 \mathrm{~J}$, or $2 \mathrm{~J} / \mathrm{L}$ of mixture. For comparison, a laboratory centrifuge that can treat up to $1.5 \mathrm{~L}$ of the acid-CNC mixture operates at 1200 Watts of power. Centrifugation for 10 minutes therefore required $720000 \mathrm{~J}$ (1200 W x $600 \mathrm{~s}$ ), which means $480000 \mathrm{~J} / \mathrm{L}$ of mixture. This comparison shows that the press separator developed in the present work was 5 orders of magnitude more energy efficient than the conventionally employed method. While the press separator required a significantly longer total operation time than the centrifuge does, its benefit is the extremely low energy input, ease of further scaling up and an inherently safer process, due to its slow movement. The press is also expected to be significantly less prone to breaking than a centrifuge operating at high speed and easier to repair. Moreover, further improvement on the press can yet make the process more effective. This work shows that the press separator concept could be developed easily for industrial production of $\mathrm{CNCs}$ and therefore will contribute to production of CNCs at a significantly lower cost, making a significantly higher overall impact of this renewable and sustainable nanomaterial. 


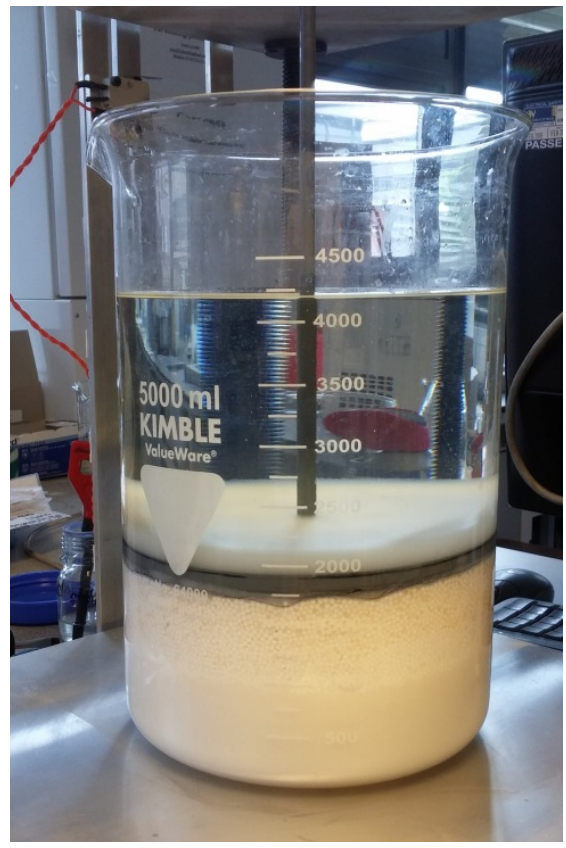

Figure 9. Press separator prototype for the acid-CNC separation in a $5 \mathrm{~L}$ sized beaker after separation. Liquid removal rate was $\mathrm{Ca} .65 \%$. Some CNCs that escaped the PS bed trap can be seen settled above the PVC piston.

\section{Conclusions}

In this study, the phase behaviour of a volume filling mixture of $12.6 \mathrm{mg} / \mathrm{ml} \mathrm{CNCs}$ in $30 \mathrm{wt} \%$ sulphuric acid was investigated theoretically for a development of a practical and energy efficient separation system. This mixture was an intermediate mixture from a CNC extraction process, which requires separation to recover both the CNCs as the product and the acid for recycling. Firstly, the volume filling behaviour was explained theoretically by a combination of the interaction energy calculation, particle distance estimation and a simple drawn model. Then, the same approach showed that the CNCs within the initially volume filling mixture can be favourably rearranged to a higher degree of compaction. In this process, the liquid acid occupying initially the same space will be removed and thereby separation was achieved. The mechanism developed to achieve this separation was based on a press system which was comprised of a particle bed for $\mathrm{CNC}$ attachment and subsequently for delivering compression to the CNC structure. The prototype press was developed on a $2 \mathrm{~L}$ scale and $5 \mathrm{~L}$ scale. A liquid removal rate of $65 \%$ was achieved in a single press process over an 8 hours and 12 hours experiment period. The liquid removal rate was the same as achieved using centrifugation at $10000 \mathrm{rpm}$ for 10 minutes. The comparison of energy efficiency between the press method developed in this work and the centrifugation method shows that the press method was 5 magnitudes of order more energy efficient than the centrifuge. We believe that this development, for its merit in ease of scaling up, excellent energy efficiency and also as an inherently safer process, will be of huge interest to the emerging industries on developing materials and applications based on cellulose nanocrystals.

\section{Acknowledgements}

We thank the UK Engineering and Physical Science Research Council (EPSRC) for funding this work through the SPACE: Sustainable Production of Aerogels from Cellulose project (Grant no. EP/J015687/1). We also thank two technicians from The University of 
Nottingham Faculty of Engineering, Karl Gregg and Alex Jackson-Crisp, for their contribution into the separator prototype fabrication.

\section{References}

[1] M. Samir; F. Alloin; A. Dufresne: Biomacromolecules, 6, (2005), 612.

[2] S. J. Eichhorn; A. Dufresne; M. Aranguren; N. E. Marcovich; J. R. Capadona; S. J. Rowan; C. Weder; W. Thielemans; M. Roman; S. Renneckar; W. Gindl; S. Veigel; J. Keckes; H. Yano; K. Abe; M. Nogi; A. N. Nakagaito; A. Mangalam; J. Simonsen; A. S. Benight; A. Bismarck; L. A. Berglund; T. Peijs: J. Mater. Sci., 45, (2010), 1.

[3] S. Kalia; A. Dufresne; B. M. Cherian; B. S. Kaith; L. Averous; J. Njuguna; E. Nassiopoulos: Int. J. Polym. Sci., (2011).

[4] A. B. Elmabrouk; T. Wim; A. Dufresne; S. Boufi: J. Appl. Polym. Sci., 114, (2009), 2946.

[5] M. Labet; W. Thielemans: Cellulose, 18, (2011), 607.

[6] N. L. G. de Rodriguez; W. Thielemans; A. Dufresne: Cellulose, 13, (2006), 261.

[7] G. Morandi; L. Heath; W. Thielemans: Langmuir, 25, (2009), 8280.

[8] A. P. Mathew; W. Thielemans; A. Dufresne: J. Appl. Polym. Sci., 109, (2008), 4065.

[9] J. R. Capadona; K. Shanmuganathan; S. Triftschuh; S. Seidel; S. J. Rowan; C. Weder: Biomacromolecules, 10, (2009), 712.

[10] Q. J. Wu; M. Henriksson; X. Liu; L. A. Berglund: Biomacromolecules, 8, (2007), 3687.

[11] L. Heath; W. Thielemans: Green Chem., 12, (2010), 1448.

[12] Y. Habibi; A. L. Goffin; N. Schiltz; E. Duquesne; P. Dubois; A. Dufresne: J. Mater. Chem., 18, (2008), 5002.

[13] D. Klemm; B. Heublein; H.-P. Fink; A. Bohn: Angew. Chem., Int. Ed., 44, (2005), 3358.

[14] M. J. Bonne; K. J. Edler; J. G. Buchanan; D. Wolverson; E. Psillakis; M. Helton; W. Thielemans; F. Marken: J. Phys. Chem. C, 112, (2008), 2660.

[15] M. J. Bonne; E. Galbraith; T. D. James; M. J. Wasbrough; K. J. Edler; A. T. A. Jenkins; M. Helton; A. McKee; W. Thielemans; E. Psillakis; F. Marken: J. Mater. Chem., 20, (2010), 588.

[16] S. Eyley; W. Thielemans: Chem. Commun., 47, (2011), 4177.

[17] L. J. Nielsen; S. Eyley; W. Thielemans; J. W. Aylott: Chem. Commun., 46, (2010), 8929.

[18] S. Y. Liew; W. Thielemans; D. A. Walsh: J. Phys. Chem. C, 114, (2010), 17926.

[19] S. Y. Liew; D. A. Walsh; W. Thielemans: Rsc Advances, 3, (2013), 9158.

[20] S. Liew; W. Thielemans; D. Walsh: J. Solid State Electrochem., (2014), 1.

[21] X. Wu; J. Tang; Y. Duan; A. Yu; R. M. Berry; K. C. Tam: J. Mater. Chem. A, 2, (2014), 19268.

[22] X. Wu; V. L. Chabot; B. K. Kim; A. Yu; R. M. Berry; K. C. Tam: Electrochim. Acta, $138,(2014), 139$.

[23] S. Elazzouzi-Hafraoui; Y. Nishiyama; J. L. Putaux; L. Heux; F. Dubreuil; C. Rochas: Biomacromolecules, 9, (2008), 57.

[24] S. Beck-Candanedo; M. Roman; D. G. Gray: Biomacromolecules, 6, (2005), 1048.

[25] X. M. Dong; T. Kimura; J. F. Revol; D. G. Gray: Langmuir, 12, (1996), 2076.

[26] D. Bondeson; A. Mathew; K. Oksman: Cellulose, 13, (2006), 171.

[27] R. Dash; Y. Li; A. J. Ragauskas: Carbohydr. Polym., 88, (2012), 789.

[28] S. Y. Liew; W. Thielemans; B. Hewakandamby: Journal of Nano Research, 38, (2016), 58.

[29] P. A. Buining; A. P. Philipse; H. N. W. Lekkerkerker: Langmuir, 10, (1994), 2106. 
[30] J. F. Revol; H. Bradford; J. Giasson; R. H. Marchessault; D. G. Gray: Int. J. Biol. Macromol., 14, (1992), 170.

[31] M. J. Sparnaay: Recueil des Travaux Chimiques des Pays-Bas, 78, (1959), 680.

[32] L. Bergström; S. Stemme; T. Dahlfors; H. Arwin; L. Ödberg: Cellulose, 6, (1999), 1.

[33] I. Kalashnikova; H. Bizot; B. Cathala; I. Capron: Langmuir, 27, (2011), 7471.

[34] I. Kalashnikova; H. Bizot; B. Cathala; I. Capron: Biomacromolecules, 13, (2012), 267.

[35] Y. Boluk; R. Lahiji; L. Zhao; M. T. McDermott: Colloids Surf., A, 377, (2011), 297. 